\title{
Osteomielite crônica multifocal recorrente da mandíbula: relato de três casos
}

\author{
Chronic recurrent multifocal osteomyelitis of the mandible: report of three cases
}

\author{
Luciana B. Paim ${ }^{1}$, Bernadete Lourdes Liphaus ${ }^{2}$, André C. Rocha ${ }^{3}$, \\ Aura Ligia Z. Castellanos ${ }^{4}$, Clovis Artur A. Silva ${ }^{5}$
}

\section{Resumo}

Objetivo: Relatar três casos de osteomielite crônica multifocal recorrente de mandíbula. A osteomielite crônica multifocal recorrente é uma doença inflamatória, com acometimento de um ou mais ossos, sem patógenos isolados nas áreas afetadas, sendo o envolvimento da mandíbula raramente descrito na literatura.

Descrição: Caso 1 - paciente feminina, com 13 anos, após tratamento dentário evoluiu com dor mandibular e febre. A paciente foi tratada com antibióticos por osteomielite, evoluindo com aumento progressivo da mandíbula e pustulose palmoplantar. A cintilografia óssea mostrou hipercaptação difusa da mandíbula. A redução da tumoração mandibular foi evidenciada com a indometacina e sessões de câmara hiperbárica. Caso 2 - paciente feminina, com 9 anos, apresentou dor e edema em região de mandíbula direita recorrente por três anos. O diagnóstico de osteomielite foi realizado e introduzido amoxicilina. Após três meses, a tomografia computadorizada mostrou osteólise mandibular difusa. Indometacina e câmara hiperbárica foram introduzidas, porém a paciente apresentou recidiva e foi tratada com prednisona, rofecoxib e metotrexato. Caso 3 paciente masculino, com 10 anos, apresentou pustulose palmoplantar e aumento difuso da mandíbula recorrente. A tomografia computadorizada de mandíbula evidenciou osteólise mandibular, e a cintilografia óssea, hipercaptação difusa. O paciente foi tratado com prednisona. O rofecoxib foi substituído após duas recidivas.

Comentários: A osteomielite crônica multifocal recorrente da mandíbula pode apresentar um curso doloroso prolongado, com intervalos de atividade e remissão do processo inflamatório. O seu reconhecimento é importante para evitar uma antibioticoterapia prolongada e procedimentos invasivos desnecessários. díbula.

\begin{abstract}
Objective: To report three cases of chronic recurrent multifocal osteomyelitis of the mandible, an inflammatory disease affecting one or more bones with absence of isolated microorganisms in affected areas.

Description: The first case is a 13 year-old female presenting with pain and fever after dental treatment. The patient received antibiotic treatment for osteomyelitis, but developed progressive enlargement of the mandible and palmoplantar pustulosis. Bone scintigraphy showed intense and diffuse uptake in the mandible. The swelling decreased after indomethacin and hyperbaric oxygen therapy. Case 2 is a 9 year-old female patient with recurrent pain and edema of the right mandible for three years. The diagnosis of osteomyelitis was established and amoxacillin introduced. After three months, tomography showed diffuse mandible osteolysis. Indomethacin and hyperbaric oxygen therapy were introduced, however the patient presented a relapse and was treated with prednisone, rofecoxib and methotrexate. Patient 3, a 10 year-old male, had palmoplantar pustulosis and recurrent enlargement of the mandible. Tomography showed diffuse mandible osteolysis and scintigraphy revealed intense and diffuse uptake in the mandible. The patient was treated with prednisone. Rofecoxib was replaced after two relapses.
\end{abstract}

Comments: Chronic recurrent multifocal osteomyelitis of the mandible is often associated with prolonged pain periods and periods of activity and remission of the inflammatory process. Its recognition is important to prevent the patient from being submitted to prolonged antibiotic therapy and unnecessary invasive procedures.

J Pediatr (Rio J) 2003;79(5):467-70: Chronic osteomyelitis, mandible.

1. Médica estagiária da complementação especializada da Unidade de Reumatologia Pediátrica do Instituto da Criança HC-FMUSP.

2. Mestre em Medicina pela FMUSP. Médica assistente da Unidade de Reumatologia Pediátrica do Instituto da Criança HC-FMUSP.

3. Mestre em Patologia Bucal pela FAOUSP. Assistente do Serviço de Bucomaxilo do Instituto de Ortopedia HC-FMUSP.

4. Médica pós-graduanda do Departamento de Pediatria da FMUSP.

5. Doutor em Medicina pela FMUSP. Chefe da Unidade de Reumatologia Pediátrica do Instituto da Criança HC-FMUSP.

Artigo submetido em 12.03.03, aceito em 28.05.03. 


\section{Introdução}

A osteomielite crônica multifocal recorrente (OCMR) é uma doença inflamatória óssea rara, com acometimento de um ou mais ossos, com períodos de exacerbação e remissão, e habitualmente sem agentes infecciosos isolados nas áreas afetadas $^{1-6}$. O envolvimento sistêmico é excepcional, e a associação com lesões cutâneas é freqüente, particularmente pustulose palmoplantar ${ }^{2,4,6}$. Os ossos mais acometidos são o esterno, a clavícula, a costelas, a coluna, a pelve e os ossos longos periféricos ${ }^{1,2}$. O acometimento isolado de mandíbula é raro, podendo ocorrer múltiplas lesões ${ }^{7}$.

As alterações radiográficas são similares às da osteomielite séptica, com lesões líticas e esclerose circunscrita. A cintilografia óssea auxilia na procura de lesões assintomáticas ${ }^{1,2}$. A biópsia geralmente não evidencia um agente infeccioso. O prognóstico é incerto, podendo apresentar um curso doloroso prolongado ${ }^{1-3}$. O tratamento é habitualmente realizado com antiinflamatórios não hormonais, corticosteróides, sulfasalazina e/ou metotrexato ${ }^{1,8,9}$.

O objetivo do presente estudo foi descrever três casos de OCMR de mandíbula acompanhados na unidade de reumatologia pediátrica do Instituto da Criança do Hospital das Clínicas da Faculdade de Medicina da Universidade de São Paulo, no período de 1998 a 2003.

\section{Descrição dos casos \\ Caso 1}

Paciente feminina, branca, com 13 anos e 3 meses, em março de 1995, após tratamento dentário, evoluiu com dor mandibular difusa, hiperemia local e febre. Recebeu diversos antibióticos (penicilina cristalina, amoxicilina, clindamicina e metronidazol) por diagnóstico de osteomielite, evoluindo com períodos de melhora e exacerbação da tumoração mandibular. Em novembro de 1998, foi internada em nosso serviço, para investigação da tumoração difusa de mandíbula, associada à limitação da abertura bucal e pustulose palmoplantar. Os exames complementares revelaram tomografia computadorizada (TC) com osteólise mandibular difusa (aspecto em vidro despolido), sem presença de coleções; biópsia de mandíbula evidenciou células gigantes com estroma de células fusiformes e infiltrado inflamatório neutrofílico, e a cultura da biópsia isolou Propionibacterium sp. A cintilografia óssea mostrou hipercaptação difusa, acentuada e heterogênea do radiofármaco em toda extensão da mandíbula, em grau menos acentuado nos ramos mandibulares. A velocidade de hemossedimentação (VHS) foi de $55 \mathrm{~mm} 1^{\mathrm{a}}$ hora e a proteína C-reativa (PCR) $80 \mathrm{mg} / \mathrm{dl}$. O hemograma completo, uréia, creatinina, coagulograma, urina I e radiografias de coluna total e ossos longos foram normais. O antígeno HLA-B27, fator antinúcleo (FAN), fator reumatóide (FR) foram negativos. As culturas de sangue e urina também foram negativas. A paciente foi tratada com roxitromicina por três meses. Apesar da antibioticoterapia instituída, a paciente evoluiu com manutenção da dor, sendo introduzida indometacina pela hipótese diagnóstica de OCMR associada à pustulose palmoplantar e iniciadas sessões de câmara hiperbárica (três sessões semanais, no total de 96 sessões). Houve melhora progressiva da dor e regressão da tumoração mandibular. Em julho de 2000, foi suspensa a indometacina, pois a paciente estava assintomática há mais de um ano, e as provas inflamatórias, negativas. Em maio de 2001, apresentou recidiva, com dor e edema local, necessitando da reintrodução da indometacina. Atualmente, mantém-se assintomática em uso de indometacina com VHS de $16 \mathrm{~mm}$ $1^{\text {a }}$ hora e PCR negativo.

\section{Caso 2}

Paciente feminina, branca, com nove anos e cinco meses, apresentou dor de forte intensidade e edema da mandíbula recorrente por três anos. Negava febre, secreção purulenta ou trauma local. A investigação odontológica não evidenciou anormalidades bucais. Em novembro de 2000, a paciente foi encaminhada ao serviço de Bucomaxilo. O exame físico inicial evidenciou tumoração dolorosa submandibular direita, sem eritema e sem calor local, com limitação da abertura da boca e gengivas levemente edemaciadas. A hipótese diagnóstica inicial foi de osteomielite, sendo realizada punção aspirativa e iniciada amoxacilina. Após três meses de tratamento, sem melhora clínica e laboratorial, foi encaminhada ao Instituto da Criança, sendo introduzido naproxeno e amoxicilina. Este último utilizado por 8 meses, até normalização do VHS. Evoluiu com aumento bilateral da mandíbula com dor à palpação e limitação da abertura da boca. Os exames complementares mostraram VHS $26 \mathrm{~mm} 1^{\mathrm{a}}$ hora, PCR 7,0 mg/dl. O hemograma completo, uréia, creatinina, coagulograma, urina I, radiografia de tórax e ossos longos foram normais. Antígeno HLA-B27, FAN, FR, culturas de sangue e urina foram negativas. A TC da mandíbula mostrou espessamento, esclerose dos ramos da mandíbula e corpo com áreas líticas arredondadas (padrão em vidro despolido), compatível com displasia óssea. O diagnóstico de OCMR foi estabelecido e introduzido indometacina e câmara hiperbárica (total de 80 sessões). Em novembro de 2001, a paciente apresentou recidiva (dor importante e aumento do volume da mandíbula). A TC evidenciou aumento volumétrico de aspecto osteodisplásico da mandíbula bilateralmente, com ligeira tumefação de partes moles adjacentes e cistos ósseos. A paciente recebeu um mês de prednisona, com melhora, e apresentou recidiva após sua suspensão, sendo necessária a utilização de rofecoxib $25 \mathrm{mg} /$ dia e metotrexato 20 $\mathrm{mg} / \mathrm{semana}$. Após dois meses, apresentou melhora clínica importante, e a VHS atual é de $32 \mathrm{~mm} 1^{\mathrm{a}}$ hora.

\section{Caso 3}

Paciente masculino, branco, com 10 anos, desde agosto de 2000 apresentou pustulose palmoplantar e aumento difuso da mandíbula bilateral com dor, calor, febre e redu- 
ção da abertura bucal, que melhoram após o uso de antiinflamatório não hormonal (diclofenaco). Em novembro de 2001, foi encaminhado aos serviço de Bucomaxilo e unidade de Reumatologia Pediátrica por recidiva da dor. Os exames complementares evidenciaram TC com espessamento, esclerose a áreas de osteólise mandibular difusa (aspecto em vidro despolido), compatível com displasia óssea; biópsia de mandíbula evidenciou processo inflamatório crônico com fibrose. A cintilografia óssea mostrou hipercaptação difusa. A VHS inicial foi de $50 \mathrm{~mm} 1^{\text {a }}$ hora e a PCR $6,0 \mathrm{mg} / \mathrm{dl}$. A radiografia panorâmica da mandíbula evidenciou lesões líticas e espessamento cortical. O hemograma completo e radiografias de coluna total e ossos longos foram normais. O antígeno HLA-B27, FAN e FR foram negativos. As culturas de sangue e urina também foram negativas. $\mathrm{O}$ diagnóstico de OCMR foi estabelecido e o paciente tratado inicialmente com prednisona por um mês. O paciente apresentou duas recidivas (janeiro e fevereiro de 2002), sendo mantido com rofecoxib $25 \mathrm{mg} /$ dia. Atualmente com regressão total da tumoração. A radiografia panorâmica da mandíbula evidenciou melhora do espessamento cortical.

\section{Discussão}

Os pacientes com osteomielite crônica multifocal recorrente apresentam início insidioso de calor, dor e edema de partes moles, restritos a um ou mais ossos ${ }^{1,3}$. Manifestações sistêmicas incluindo febre e perda de peso ocorrem habitualmente na fase aguda da doença ${ }^{1,2}$, como ocorreu em dois dos nossos pacientes.

O acometimento é usualmente simétrico e multifocal (podendo apenas um osso ser acometido). O curso é intermitente, com períodos de remissão e exacerbação ${ }^{2,3}$. Os ossos dos membros inferiores são os mais acometidos (55\%), seguidos pelo esqueleto axial (pelve em 55\%, coluna em $15 \%$, parede torácica em $13 \%$ ) e clavículas $(8 \%)^{1}$.

Raramente a doença pode afetar isoladamente a mandíbula, podendo ocorrer lesões múltiplas ${ }^{7}$, como observado em nossos pacientes. Benedetta et al. ${ }^{9}$ descreveram 260 pacientes pediátricos com OCMR, e o envolvimento de mandíbulas ocorreu em apenas 13 pacientes (5\%).

A OCMR, na faixa etária pediátrica, pode estar associada a várias manifestações cutâneas: pustulose palmoplan$\operatorname{tar}^{1-3}$ (considerada por alguns dermatologistas como variante da psoríase vulgaris $)^{5}$, pustulose difusa, psoríase vulgaris, acne ${ }^{9-11}$, síndrome de Sweet e pioderma gangrenoso $^{9}$. Dois dos nossos pacientes apresentaram pustulose palmoplantar.

As provas de fase aguda (VHS e PCR) estão elevadas nas fases ativas e normalizam nas remissões ${ }^{4}$. As culturas são habitualmente negativas para bactérias, fungos e micobactérias no material ósseo aspirado, no entanto o Propionibacterium acnes pode estar associado à $\mathrm{OCMR}{ }^{1,9,11}$. As radiografias mostram uma destruição lítica em região me- tafisária de ossos longos, seguida por esclerose progressiva. A cintilografia óssea é útil na detecção de lesões silenciosas, mostrando aumento de captação nas áreas afetadas $^{1,2}$. A ressonância nuclear magnética ou a tomografia computadorizada, apesar de não serem essenciais ao diagnóstico, são úteis, para mostrar a extensão das lesões e o envolvimento de articulações e de tecidos moles adjacentes $^{1}$.

A biópsia evidencia que a lesão não é séptica e que este agente funciona como um desencadeante de reação imunológica e inflamatória ${ }^{16}$, como observado no caso 1 . O estudo histopatológico da lesão óssea é variável. A lesão inicial é caracterizada pela presença de neutrófilos, recebendo a denominação de pseudo-abscesso. A lesão crônica demonstra predominância de linfócitos, com ocasional presença de plasmócitos, histiócitos e fibrose ${ }^{1,9}$.

Estudos recentes sugerem os seguintes critérios para diagnóstico da OCMR: doença com duração acima de três meses; evidência histológica de inflamação óssea crônica, excluindo outras doenças e ausência de crescimento bacteriano nas culturas 9 .

Alguns autores consideram a OCMR como parte do espectro da síndrome SAPHO (sinovite, acne, pustulose palmoplantar, hiperostose e osteólise). Esse acrônimo enfatiza a associação entre inflamação óssea e manifestações cutâneas $^{9-11}$. Descreve-se uma associação da síndrome SAPHO com as espondiloartropatias e o antígeno HLAB27. A prevalência do antígeno HLA-B27 é de 9\% nos pacientes pediátricos europeus com $\mathrm{OCMR}^{9}$, particularmente nos pacientes pediátricos com lesões ósseas e cutâneas concomitantes ${ }^{1}$. Os nossos três pacientes não apresentavam o antígeno HLA-B27.

Outros autores denominam a OCMR de localização exclusiva em mandíbula como osteomielite esclerosante difusa da mandíbula $(\mathrm{OEDM})^{12-14}$. Devido à semelhança clínica, radiológica, cintilográfica e histológica com a síndrome SAPHO, tem sido levantada a hipótese de que a OEDM possa ser uma manifestação localizada da mesma entidade $^{12}$.

O uso de terapia antimicrobiana para a OCMR não altera o curso da doença ${ }^{4}$. Os antiinflamatórios não hormonais (naproxeno, indometacina ou aspirina) constituem a terapêutica inicial, sendo indicado um curso rápido de corticosteróides nos casos refratários. Os antiinflamatórios não hormonais inibidores da ciclo-oxigenase 2 , como o meloxicam, têm sido utilizados nos casos de OCMR não responsivos ao naproxeno ${ }^{6}$. Dois de nossos casos usaram rofecoxib na dose de $0,6 \mathrm{mg} / \mathrm{kg} / \mathrm{dia}$, conforme a dose utilizada previamente na artrite reumatóide juvenil ${ }^{15}$. Outros tratamentos, tais como, sulfasalazina ${ }^{1}$, metotrexato ${ }^{8} \mathrm{e}$ câmara hiperbárica ${ }^{12}$ são indicados nos casos refratários e dolorosos, como foi necessário utilizar em dois dos nossos casos.

A câmara hiperbárica ou oxigenação hiperbárica é um procedimento seguro e eficaz nos casos de osteomielites 
agudas e crônicas, podendo ser utilizada nos pacientes com dor intensa e mantida ${ }^{12,16}$. O oxigênio hiperbárico é cicatrizante, estimulando os fibroblastos, aumentando a produção de colágeno e a angiogênese ${ }^{16}$.

O prognóstico da OCMR é duvidoso, podendo apresentar um curso doloroso prolongado, com intervalos de atividade e remissão do processo inflamatório ${ }^{1-3}$.

O reconhecimento da OCMR pelos pediatras é importante, devendo diferenciar da osteomielite aguda e evitar antibioticoterapia prolongada desnecessária. A presença de tumorações mandibulares dolorosas recorrentes ou crônicas (acima de três meses) alerta para o diagnóstico. A cintilografia, tomografia ou ressonância são úteis para identificar as lesões líticas. A biópsia é necessária para afastar tumor ósseo e osteomielite infecciosa. O tratamento inicial inclui antiinflamatórios não hormonais e/ou corticosteróides. A câmara hiperbárica ou metotrexato podem ser utilizados nos casos refratários, com dores mantidas ou importantes.

\section{Referências bibliográficas}

1. Job-Deslandre C, Krebs S, Kahan A. Chronic recurrent multifocal osteomyelitis: five-years outcomes in 14 patients cases. J Bone Spin 2001;64:245-51.

2. Schultz C, Holterhus PM, Seidel A, Jonas S, Barthel M, Kruse K, et al. Chronic recurrent multifocal osteomyelitis in children. Pediatr Infect Dis J 1999;18:1008-13.

3. Bjorkstén B, Gustavson KH, Eriksson B, Lindholm A, Nordstrom S. Chronic recurrent multifocal osteomyelitis and pustulosis palmoplantaris. J Pediatr 1978;93:227-31.

4. King SM, Laxer RM, Manson D, Gold R. Chronic recurrent multifocal osteomyelitis: a noninfectious inflammatory process. Pediatr Infect Dis J 1987;6:907-11.

5. Ravelli A, Marseglia GL, Viola S, Ruperto N, Martini A. Chronic recurrent multifocal osteomyelitis: with usual features. Acta Paediatr 1995;84:222-5.

6. Girschick HJ, Raab P, Kirschner S, Suerbaum S, Wurzburg T. Chronic recurrent multifocal osteomyelitis in children: long term follow-up and treatment of relapses. Arthritis Rheum 2001;44 Suppl 9:272.

7. Lavis JF, Gigon S, Gueit I, Michot C, Tardif A, Mallet E, et al. Chronic recurrent multifocal osteomyelitis of the mandible. A case report. Arch Pediatr 2002;9:1252-5.
8. Akikusa JD, Zacharin M, Shugg AW, Melbourne RCA, Tasmania VC. Bisphosphonates in the treatment of chronic recurrent multifocal osteomyelitis. Arthritis Rheum 2001;44 Suppl 9:171.

9. Beretta-Piccoli BC, Sauvain MJ, Gal I, Saurenmann AST, Kressebuch H, Bianchetti MG. Synovitis, acne, pustulosis, hyperostosis, osteitis (SAPHO) syndrome in childhood: a report of ten cases and review of the literature. Eur J Pediatr 2000;159:594-601.

10. Hamanova J, Kabicek P. The SAPHO syndrome in a 16-year-old boy: coincidence of acne conglobata and osteoarthritis. J Adolesc Health 1993;14:120-3.

11. Kotilainen P, Merilahti-Palo R, Lehtonen OP, Manner I, Helander I, Möttönen T, et al. Propionibacterium acnes isolated from sternal osteitis in a patient with SAPHO syndrome. J Rheum 1996;23:1302-4.

12. van Merkesteyn JPR, Groot RH, Bras J, McCarroll RS, Bakker DJ. Diffuse sclerosing osteomyelitis of the mandible: A new concept of its etiology. Oral Surg Oral Med Oral Pathol 1990;70(4):414-9.

13. Suei Y, Taguchi A, Tanimoto K. Diffuse sclerosing osteomyelitis of the mandible: its characteristics and possible relationship to synovitis, acne, pustulosis, hyperostosis, osteitis (SAPHO) syndrome. J Oral Maxillofac Surg 1996;54:1194-1200.

14. Kahn MF, Hayem F, Hayem G, Grossin M. Is diffuse sclerosing osteomyelitis of the mandible part of the synovitis, acne, pustulosis, hyperostosis, osteitis (SAPHO) syndrome? Analysis of seven cases. Oral Surg Oral Med Oral Pathol 1994;78:594-8.

15. Rose ES, Ferrandiz M, Kiss M, Forre O, Vehe R, Higgins G, et al. Steady-state plasma concentrations of rofecoxib in children (ages 2-5 years) with juvenile rheumatoid arthritis. Arthritis Rheum 2001;44 Suppl:291.

16. Jon T, Kenneth R, Adams M, William R. Efeitos fisiológicos do oxigênio hiperbárico. J Trauma 1993;15:1-3.

Endereço para correspondência:

Dr. Clovis Artur Almeida da Silva

Rua Raul Pompéia, 303/43 - Pompéia

CEP 05025-010 - São Paulo - SP

Tel.: (11) 3865.1901 - Fax: (11) 3069.8503

E-mail: clovisaas@icr.hcnet.usp.br 